\title{
Stability of minimal surfaces in spaces of constant curvature
}

J. L. Barbosa and M. do Carmo

\section{Introduction.}

1.1. Let $x: M \rightarrow \bar{M}^{n}, n \geq 3$, be a minimal immersion of a $C^{3}$ 2-dimensional orientable manifold $M$ into an $n$-dimensional smooth Riemannian manifold $\bar{M}^{n}$. Let $D \subset M$ be a domain with compact closure $D$ and piecewise smooth boundary $\partial D$. Call $D$ stable if it is a relative minimum for the area function of the induced metric for all variations that leave $\partial D$ fixed.

In a previous paper [1] we described the idea of a method to obtain sufficient conditions for the stability of $D$ and applied it to the cases where $\bar{M}^{n}$ is the 3-sphere $S^{3}(a)$ with constant curvature $a>0$, the 3 -hyperbolic space $H^{3}(a)$ with constant curvature $a<0$, and the euclidean space $R^{n}$, $n \geq 3$. In this paper, we present a more detailed description of the method and apply it to the case where the ambient space is a Riemannian manifold with constant sectional curvature a. The results are as follows ( $K$ will always denote the Gaussian curvature of $M$ in the induced matric).

1.2 Theorem. Let $x: M \rightarrow \Delta^{n}(a)$ be a minimal immersion, where $\Delta^{n}(a)$ is either $S^{n}(a)$, if $a>0$ or $H^{n}(a)$. if $a<0$. Set $c_{n}=3-(2 /(n-2))$, and assume that $D \subset M$ is simply-connected.,Then:

(i) if $a>0$ and $\int_{D}(2 a-K) d M<\frac{8 \pi}{2 c_{n}+2}, D$ is stable,

(ii) if $a<0$ and $\int_{D}|K| d \dot{M}<\frac{8 \pi}{2 c_{n}+2}$, D is stable.

Actually, Theorem 1.2 is, modulo a curvature estimate, a particular case of the following general theorem.

Let $x: M \rightarrow \bar{M}^{n}, n \geq 3$, be a minimal immersion. Assume that the sectional curvature of $\bar{M}^{n}$ is bounded above by $a$ constant $a$ and set $a^{+}=\max (a, 0)$. Let $d s^{2}$ be the metric induced by $x$ on $M$ and assume that the Gaussian curvature $\hat{K}$ of the (possibly degenerate) metric $d \sigma^{2}=\left(2 a^{+}-K\right) d s^{2}$ satisfies $\hat{K} \leq c$. Let $D \subset M$ be a simply-connected domain. Then:

(i) If $\bar{M}^{n}$ is analytic and

$$
\int_{D}\left(2 a^{+}-K\right) d M<\frac{8 \pi}{2 c+2},
$$

Recebido em dezembro de 1979. 
$D$ is stable.

(ii) If $a \neq 0$, and $\int_{D}\left(2 a^{+}-K\right) d M<\frac{8 \pi}{4 c+2}$, $D$ is stable.

Since we will show in Sec. 2 that if $\bar{M}^{n}$ is a space of constant curvature $a \neq 0$, the curvature $\hat{K} \leq c_{n}=3-\frac{2}{n-2}$, Theor. 1.2 and Theor. 1.4 below will follow from Theor. 1.3.

1.4 Theorem. Theor. 1.2 holds for minimal immersions $x: M \rightarrow \vec{M}^{n}(a)$ into any $n$ dimensional Riemannian manifold of constant curvature a provided we replace the bound $\frac{8 \pi}{2 c_{n}+2}$ by $\frac{8 \pi}{4 c_{n}+2}$.

1.5 Remark. Theor. 1.3 reduces the problem of finding a simple sufficient condition for stability to the problem of finding an estimate for the curvature $\hat{K}$ that is independent of the immersion $x$ (See Remark 4.4.).

1.6 Remark. Theor. 1.2 generalizes Theors. 1.2 and 1.3 of [1]. however, the bound in Theor. 1.2 for $a>0$ and $n>3$ is probably not sharp. Theorem 1.3 can be used, together with the estimate 2.9 of [1], to prove Theor. 1.4 of [1].

\section{Curvature estimates.}

2.1. Let $\bar{M}^{n}(a)$ be an $n$ dimensional Riemannian manifold with constant curvature $a$. In this Section we estimate the Gaussian curvature $\vec{K}$ of a metric $d \sigma^{2}$ on $M$ obtained by a conformal transformation of the metric $d s^{2}$ which is induced by a minimal immersion $x: M \rightarrow \bar{M}^{n}(a)$. More precisely, we prove the following result.

2.2 Proposition. Let $x: M \rightarrow \bar{M}^{n}(a)$ be a minimal immersion, $K$ the Gaussian curvature of the induced metric $d s^{2}$, and set $d \sigma^{2}=u d s^{2}$, where:

$$
\left\{\begin{array}{l}
u=2 a-K, \text { if } a>0 \\
u=-K, \text { if } a<0
\end{array}\right.
$$

Then the Gaussian curvature $\hat{K}$ of $d \sigma^{2}$.satisfies

$$
\hat{K} \leq c_{n}=3-\frac{2}{n-2}
$$


Proof: Let us choose an adapted orthonormal frame $e_{1}, e_{2}, e_{3}, \ldots, e_{n}$ to $x$ in $\bar{M}^{n}(a)$, that is, $e_{1}, e_{2}$ are tangent to $x(M) \subset \bar{M}^{n}(a)$, and let us agree in the following range of indices:

$$
i, j, k, \ldots=1,2, \quad \alpha, \beta, \gamma, \ldots=3, \ldots, n, \quad A, B, Z, \ldots=1, \ldots, n .
$$

We will denote by $h_{i a j}$ the coefficients of the second fundamental form of $x$ in the normal direction $e_{\alpha}$ and will write $\|B\|^{2}=2 a-2 K$, hence (2.3) can be written as

$$
u=|a|+\frac{1}{2}\|B\|^{2}>0 .
$$

It is easily computed the curvature $\hat{K}$ of $d \sigma^{2}=u d s^{2}$ is given by (Cf. e.g. [2])

$$
\hat{K}=\frac{1}{u} K+\frac{1}{2 u^{2}}\left(-\Delta u+\frac{1}{u}\|\nabla u\|^{2}\right),
$$

where $\Delta u$ is the Laplacian of $u$ and $\nabla u$ is the gradient of $u$, both computed in the metric $d s^{2}$.

From $(2.5)$, it follows that

$$
d u=d\left(|a|+\frac{1}{2} \sum_{i \alpha j} h_{i \alpha j}^{2}\right)=\sum_{i \alpha j k} h_{i \alpha j} h_{i \alpha j k} \omega_{k}
$$

where $\omega_{A}$ is the pull-back by $x$ of the coframe $\theta_{A}$ of $e_{A}$ (since $e_{A}$ is an adapted frame, $\omega_{\alpha}=0$ ), and $h_{i \alpha j k}$ are the components of the covariant derivative of the second fundamental form of $x$. Thus the components of the gradient $\nabla u$ of $u$ are

$$
u_{k}=\sum_{i \alpha j} h_{i \alpha j} h_{i \alpha j k}
$$

By taking the covariante derivative $D$ of $\nabla u$, we obtain

$$
D\left(u_{k}\right)=\sum_{i \alpha j \ell .} h_{i \alpha j \ell} h_{i \alpha j k} \omega_{\ell}+\sum_{i \alpha j \ell} h_{i \alpha j} h_{i \alpha j k \ell} \omega_{\ell}
$$

where $h_{i \alpha j k \ell}$ are the components of the second covariant derivative of $h_{i \alpha j}$. Thus, the componentes of $D(\nabla u)$ are

$$
u_{k \ell}=\sum_{i a j} h_{i \alpha j \ell} h_{i a j k}+\sum_{i a j} h_{i \alpha j} h_{i \alpha j k \ell}
$$


and the Laplacian of $u$ is given by

$$
\Delta u=\sum_{k} u_{k k}=\sum_{i a j k} h_{i \alpha j k}^{2}+\sum_{i \alpha j} h_{i \alpha j} \Delta_{h i \alpha j}
$$

where $\Delta h_{i a j}$ is the Laplacian of the second fundamental form. We now use Simons inequality ([3], pg. 41)

$$
-\sum_{i \alpha j} h_{i \alpha j} \Delta h_{i \alpha j} \leq\left(2-\frac{1}{n-2}\right)\|B\|^{4}-2 a\|B\|^{2}
$$

to estimate the second summand of the right hand side of (2.8), and by (2.5)-(2.9), we obtain

$$
\begin{aligned}
\hat{K} & \leq \frac{K}{u}+\frac{2}{u^{2}}\left\{\left(2-\frac{1}{n-2}\right)(u-|a|)^{2}-a(u-|a|)\right\}+ \\
& +\frac{1}{2 u^{3}}\left\{-\left(|a|+\frac{1}{2} \|\left. B\right|^{2}\right) \sum_{i \alpha j k} h_{i \alpha j k}^{2}+\sum_{k}\left(\sum_{i \alpha j} h_{i a j} h_{i \alpha j k}\right)^{2}\right\}
\end{aligned}
$$

We need the following lemma.

2.11 Lemma. $W=-\frac{1}{2}\|B\|^{2} \sum_{i \alpha j k} h_{i \alpha j k}^{2}+\sum_{k}\left(\sum_{i \alpha j} h_{i \alpha j} h_{i \alpha j k}\right)^{2} \leq 0$.

Proof of the Lemma. Since $\|B\|^{2}=\sum_{r \beta s} h_{r \beta s}^{2}$, we have

$$
W \leq-\frac{1}{2} \sum_{r \beta s} h_{r \alpha s}^{2} \sum_{i \alpha j k} h_{i \alpha j k}^{2}+\sum_{k}\left(\sum_{i \alpha j} h_{i \alpha j} h_{i \alpha j k}\right)^{2}=T .
$$

Thus it suffices to prove that $T=0$. Actually we will prove that this is so for each fixed $\alpha$.

By minimality, and the fact that the ambient space has constant curvature, we have, for each $\alpha$, that $h_{1 \alpha 1}=-h_{2 \alpha 2}, h_{i \alpha j j}=-h_{i \alpha k k}$ and $h_{i \alpha j k}$ is symmetric in latin indices. Thus, on the one hand,

$$
\sum_{r s i j k} h_{r a s}^{2} h_{i \alpha j k}^{2}=8\left(h_{1 \alpha 1}^{2}+h_{1 \alpha 2}^{2}\right)\left(h_{1 \alpha 11}^{2}+h_{1 \alpha 12}^{2}\right)
$$

and on the other hand, 


$$
\begin{gathered}
\sum_{k}\left(\sum_{i j} h_{i \alpha j} h_{i \alpha j k}\right)^{2}=\sum_{i j}\left(h_{i \alpha j} h_{i \alpha j 1}\right)^{2}+\left(\sum_{i j} h_{i \alpha j} h_{i \alpha j 2}\right)^{2}= \\
=4\left(h_{1 \alpha 1} h_{1 \alpha 11}+h_{1 \alpha 2} h_{1 \alpha 12}\right)^{2}+4\left(h_{1 \alpha 1} h_{1 \alpha 12}-h_{1 \alpha 2} h_{1 \alpha 11}\right)^{2}= \\
=4\left(h_{1 \alpha 1}^{2}+h_{1 \alpha 2}^{2}\right)\left(h_{1 \alpha 11}^{2}+h_{1 \alpha 12}^{2}\right) .
\end{gathered}
$$

It follows that $T=0$ and this proves the Lemma.

To prove that $\hat{K} \leq c_{n}$, we will consider two cases. obtain

First, let $a<0$. Then $u=-K$. By (2.10) and Lemma (2.11), we

$$
\begin{aligned}
\hat{K} & \leq-1+\frac{2}{u^{2}}\left\{\left(1+\frac{n-3}{n-2}\right)(u+a)^{2}-a(u+a)\right\}= \\
& =-1+2\left(\frac{u+a}{u}\right)+2\left(\frac{n-3}{n-2}\right)\left(\frac{u+a}{u}\right)^{2} \leq \\
& \leq-1+2+2\left(\frac{n-3}{n-2}\right)=3-\frac{2}{n-2}=c_{n},
\end{aligned}
$$

and this proves Prop. 2.2 for the case $a<0$.

Now consider $a>0$. Then $u=2 a-K$. By (2.10), Lemma (2.11), and the fact that $\frac{K}{2 a-K}=\frac{2 a-u}{u}$, we obtain

$$
\hat{K} \leq \frac{2 a-u}{u}+\frac{1}{2 u^{2}}\left\{\left(2-\frac{1}{n-2}\right)(2 u-2 a)^{2}-2 a(2 u-2 a)\right\}=
$$

(2.12) $=f(u)$.

Now set $1 /(n-2)=\alpha$ and observe that

$$
f(u)=\frac{(3-2 \alpha) u^{2}-4(2-\alpha) a u+(6-2 \alpha) a^{2}}{u^{2}}
$$

By noticing that $u \geq a>0, f(a)=1, f^{\prime}(a)<0, \lim _{n \rightarrow \infty} f(u)=3-2 \alpha$ and that $f^{\prime}(u)=0$ if and only if $u=(3-\alpha) a /(2-\alpha)^{n}$, we see that $f(u) \leq$ $\leq 3-2 \alpha=c_{n}$. From (2.12), $\hat{K} \leq c_{n}$, and this concludes the proof of Prop. 2.2. 


\section{Proof. Theorem 1.2.}

3.1. We first fix our notation and make some general remarks.

Let $x: M \rightarrow \vec{M}^{n}$ be a minimal immersion into an $n$-dimensional Riemannian manifold. Choose an adapted orthonormal frame $e_{A}$ in a neighborhood of $\bar{M}^{n}$ and take the range of indices as in (2.4). Consider a domain $D \subset M$ and denote by $V=\sum_{\alpha} V_{\alpha} e_{\alpha}$ a normal vector field that vanishes on $\partial D$. Then the formula for the second variation along $V$ is (See [3])

$$
I(V, V)=\int_{D}\left(-\sum_{\alpha} V_{\alpha} \Delta V_{\alpha}-\sum_{\alpha \beta}\left(\tilde{R}_{\alpha \beta}+\sigma_{\alpha \beta}\right) V_{\alpha} V_{\beta}\right) d M
$$

Here $\bar{R}_{A B C D}$ is the curvature tensor of the Riemannian manifold $\bar{M}^{n}$, $\tilde{R}_{\alpha \beta}=\sum_{i} \bar{R}_{i \alpha i \beta}, \Delta V_{\alpha}$ are the components of the Laplacian of $V$, and

$$
\sigma_{\alpha \dot{\beta}}=\sum_{i j} h_{i \alpha j} h_{i \beta j}
$$

where $h_{i \alpha j}$ are the coefficients of the second fundamental form of $x$ in the direction $e_{\alpha}$.

$V$ is called a Jacobi field if, for each $\alpha$,

$$
-\Delta V_{\alpha}-\sum_{\beta}\left(\tilde{R}_{\alpha \beta}+\sigma_{\alpha \beta}\right) V_{\beta}=0
$$

If $\bar{M}^{n}$ is a real analytic Riemannian manifold, it follows that the minimal immersion $x$ is real analytic. As solutions of (3.2), Jacobi fields are then real analytic normal vector fields.

3.3. Now assume that $\bar{M}^{n}$ is real analytic and that $D \subset M$ is not stable. By Smale's version of the Morse index theorem ([4], [5]), there exists a domain $D^{\prime} \subset D$ and a Jacobi field $V$ that vanishes on $\partial D^{\prime}$. Since $V$ is analytic, it only vanishes along analytic curves and at isolated points. We assume that $\partial D^{\prime}$ is the "first" conjugate boundary, that is, that $V$ only vanishes in $D^{\prime}$ at isolated points $q_{1}, \ldots, q_{m}$. Away from such points, we can choose the adapted frame $e_{A}$ so that $V=u e_{3}, u>0$. This defines a function $u$ in $\dot{W}=D^{\prime}-\bigcup_{s=1}^{m}\left\{q_{s}\right\}$, where the 3-component of Eq. (3.2) is given by

$$
\Delta u+u\left\langle\Delta e_{3}, e_{3}\right\rangle+u \sum_{i} \bar{R}_{i 3 i 3}+u \sum_{i j} h_{i 3 j}^{2}=0
$$

Set $u=0$ if $V=0$. Thus $u$ is a nonnegative function in $\bar{D}^{\prime}$ that is differentiable in $W$, satisfies (3.4) in $W$, and vanishes on $\partial D^{\prime}$. Notice that $u^{2}$ is differentiable in all of $\bar{D}^{\prime}$. 
By noticing that

$$
\begin{gathered}
\left\langle\Delta e_{3}, e_{3}\right\rangle=-\sum_{i}\left|\nabla_{e_{i}}^{N} e_{3}\right|^{2}, \\
\sum_{i j} h_{i 3 j}^{2}=\sum_{i, \alpha, j} h_{i \alpha j}^{2}-\sum_{i, j, \alpha>3} h_{i \alpha j}^{2},
\end{gathered}
$$

and by setting

$$
A^{2}=\sum_{i}\left|\nabla_{e_{i}}^{N} e_{3}\right|^{2}+\sum_{i, j, \alpha>3} h_{i \alpha j}^{2}
$$

we can write (3.4) as

$$
\Delta u+u \sum_{i} \bar{R}_{i 3 i 3}+u \sum_{i \alpha j} h_{i a j}^{2}-A^{2} u=0 .
$$

3.6. Proof of Theor. 1.2. (i) Since $S^{n}(a)$ is real analytic, the assumption that $D$ is not stable implies the existence of a domain $D^{\prime} \subset D$ and a function $u: \bar{D}^{\prime} \rightarrow R$ satisfying the conditions described in 3.3. Furthermore, since the ambient space has constant curvature $a$, we have $\bar{R}_{i 3 i 3}=a$, and

$$
\sum_{i a j} h_{i a j}^{2}=2 a-2 K
$$

Thus (3.5) can be written as

$$
\Delta u+2(2 a-K) u-A^{2} u=0 .
$$

The proof is now similar to the proof of Theor. 1.4 in [1]. Consider the first eigenvalue $\lambda_{1}$ for the problem

$$
\Delta f+\lambda(2 a-K) f=0 .
$$

We claim that (3.7) implies that $\lambda_{1} \leq 2$. To see that, cover the points $q_{\mathrm{s}} \in D^{\prime}$ (See 3.3) be small nonoverlapping disks $D_{\varepsilon}\left(q_{s}\right)$ around $q_{s}$, set $D_{\varepsilon}=\bigcup_{\zeta} D_{\varepsilon}\left(q_{s}\right)$ and obtain from (3.7)

$$
\begin{gathered}
2 \int_{D^{\prime}-D_{\varepsilon}}(2 a-K) u^{2} d M \geq-\int_{D^{\prime}-D_{\varepsilon}} u \Delta u d M= \\
=\int_{D^{\prime}-D_{\varepsilon}}\|\nabla u\|^{2} d M+\frac{1}{2} \int_{\partial D_{\varepsilon}}\left\langle\nabla u^{2}, n\right\rangle d s,
\end{gathered}
$$


where $n$ is a unit normal vector along the boundary $\partial D_{\varepsilon}$. Since $u^{2}$ is differentiable, we obtain, as $\varepsilon \rightarrow 0$,

$$
2 \geq \frac{\int_{D^{\prime}}\|\nabla u\|^{2} d M}{\int_{D^{\prime}}(2 a-K) u^{2} d M} \geq \lambda_{1},
$$

as we claimed.

Now introduce in $M$ a metric $d \sigma^{2}=(2 a-K) d s^{2}$, where $d s^{2}$ is the metric induced by $x$. By Prop. 2.2, the Gaussian curvature $\hat{K}$ of $d \sigma^{2}$ satisfies $\hat{K} \leq c_{n}=3-\frac{2}{n 2}$. From Prop. 3.13 of [1], we conclude that $\lambda_{1} \geq$ $\geq \lambda_{1}\left(D^{*}\right)$, where $\lambda_{1}\left(D^{*}\right)$ is the first eigenvalue of the Laplacian of a geodesic disk in a sphere $S^{2}\left(1 / \sqrt{c_{n}}\right)$ with curvature $c_{n}$ such that

$$
A^{*}=\operatorname{area}\left(D^{*}\right)=\int_{D}(2 a-K) d M<\frac{8 \pi}{2 c_{n}+2} .
$$

By using Prop. 3.10 of [1], we compute that, for such a domain,

$$
\lambda_{1}\left(D^{*}\right) \geq \frac{2}{c_{n}} \frac{4 \pi-A^{*}}{A^{*}}>2
$$

Thus

$$
2 \geq \lambda_{1} \geq \lambda_{1}\left(D^{*}\right)>2
$$

This is a contradiction, and proves Theor. 1.2 (i).

(3.9) Proof of Theorem 1.2. (ii) Since $a<0$, it follows from (3.7) that

$$
\Delta u-2 K u \geq 0 \text {. }
$$

Now the proof follows in a way entirely similar to the proof of (i), by replacing $2 a-K$ by $-K$.

\section{Proof of Theorem 1.3.}

4.1. Suppose that $D \subset M$ is not stable. By assuming that $\bar{M}^{n}$ is real analytic (Sec. 3.3) and that the sectional curvature of $\bar{M}^{n}$ is bounded above by a, we obtain that 


$$
\sum h_{i x j}^{2}=2 \bar{R}_{1212}-2 K \leq 2 a-2 K
$$

and that $\sum_{i} \bar{R}_{i 3 i 3} \leq 2 a$. Thus (3.5) implies that

$$
\Delta u+2(2 a-K) u \geq 0, \quad \text { in } \quad W,
$$

where $W$ is defined in Sec. (3.3).

If $\bar{M}^{n}$ is not analytic, (4.2) holds for the open set of points $U \subset D^{\prime}$ where $u \neq 0$ (See definition of $D^{\prime}$ in (3.3)). Since there is a finite member of conjugate boundaries in $D^{\prime}$, we can assume that $\partial D^{\prime}$, is the first such boundary, and thus that $U$ is connected. We also can assume that $u \geq 0$ on $D^{\prime}$. Since $u^{2}$ is still differentiable in $D^{\prime}$ and in $U$

$$
\Delta u^{2}=2 u \Delta u+2\|\nabla u\|^{2},
$$

we can write (4.2) in the form

$$
\frac{1}{2} \Delta u^{2}+2 u^{2}(2 a-K)-\|\nabla u\|^{2}=u \Delta u+2 u^{2}(2 a-K) \geq 0, \text { in } U .
$$

Thus, by setting $f=u^{2}$, we see, by a limiting process, that, in $D^{\prime}$

$$
\Delta f+4 f(2 a-K) \geq 0 .
$$

In both cases (4.2) or (4.3), we obtain, by using the arguments in the proof of Theorem 1.2 (See (3.6)) that the first eigenvalue $\lambda_{1}$ of the problem

$$
\Delta g+\lambda\left(2 a^{+}-K\right) g=0, \quad a^{+}=\max (a, 0),
$$

satisfies $\lambda_{1} \leq \alpha$, where $\alpha$ is equal to 2 or 4 according to $\bar{M}^{n}$ is real analytic or not, respectively.

We now observe that the metric $d \sigma^{2}=\left(2 a^{+}-K\right) d s^{2}$ only degenerates if $a=0$. In the analytic case, this happens only at a finite number of points. We then use Prop. 3.13 and Cor. 3.20 of [1] together with Prop. 3.10 of [1] to show, in the same way as we did in the proof of Theor. 3.1, that the conditions $\hat{K} \leq c$ and

$$
\int_{D^{\cdot}}\left(2 a^{+}-K\right) d M<\frac{8 \pi}{2 c+2}
$$

imply that $\lambda_{1}>2$, a contradiction. In the nonanalytic case, we restrict ourselves to the case $a \neq 0$ and use Props. 3.13 and 3.10 of [1] to show that the conditions $\hat{K} \leq c$ and 


$$
\int_{D^{\prime}}\left(2 a^{+}-K\right) d M<\frac{8 \pi}{4 c+2}
$$

imply that $\lambda_{1}>4$, a contradiction. This completes the proof of Theor. 1.3.

4.4 Remark. Theorem 1.3 raises the question of finding an estimate for $\hat{\hat{K}}$ that depends only on bounds for geometric quantities in a general manifold $M^{n}$ (for instance, bounds for the curvature tensor of $M^{n}$ and some of its covariant derivates). From the proof of Prop. 2.2, we see that the search for such an estimate can be reduced to suitable generalizations of Lemma 2.11 and Simon's estimate 2.9).

\section{References}

[1] J. L. Barbosa and M. do Carmo, Stability of minimal surfaces and eigenvalues of the Laplacian, to appear.

[2] J. L. Barbosa and M. do Carmo, $A$ necessary condition for a metric in $M^{n}$ to be isometrically and minimally immersed in $R^{n+1}$, to appear in An. Acad Brasil Ci.

[3] S. S. Chern, Minimal submanifolds of a Riemannian manifold, University of Kansas, Dept. of Math., Tech. Rep. 19, Lawrence, Kansas, 1968.

[4] J. Simons, Minimal varieties in Riemannian manifolds, Ann. of Math, 88 (1968), 62-105.

[5] S. Smale, On the Morse index theorem, Journal of Math. and Mech, 14 (1965), 1049-1056.

J. L. Barbosa

Centro de Ciências Exatas

Departamento de Matemática

UFCE

Campos do Pici

60.000 - Fortaleza, CE

Brasil
M. do Carmo

Instituto de Matemática Pura e Aplicada

Luis de Camões, 68

20.060 - Rio de Janeiro, RJ

Brasil 